\section{Synthesis and Activity of Benzotriazolium Cephalosporins}

\author{
Jae Chul Jung, Myoung Goo Kim, \\ Moo Je Sung, Yong KeY ChoI, \\ Sang Geun An, Sea Han Oh, \\ SEONG Soo Yim and CHI JANG Moon*
}

R \& D Center, Dae Woong Pharmaceutical Co., Ltd., 223-23 Sangdaewon-Dong, Sungnam, Kyonggi-Do 462-120, Korea

(Received for publication January 31, 1995)

$\beta$-Lactam antibiotics are still among the most beneficial compounds for the treatment of often otherwise fatal infections. Aminothiazolyl cephalosporins having 3'quarternary ammonium moiety, such as cefpirome and cefepime, ${ }^{1,2)}$ show better antibacterial activity against Gram-positive and Gram-negative bacteria, including Pseudomonas aeruginosa, than that of the third generation cephalosporins. ${ }^{3)}$

Recently, it was reported that aminothiadiazolyl and aminothiazolyl cephalosporins having the delocalization of the positive charge of the condensed-heterocyclic azolium moiety ${ }^{4,5)}$ lead to an expanded antibacterial activity. In this paper, we wish to describe the synthesis and the antibacterial activity of $7 \beta$-[2-(2-aminothiazol4-yl)-2(Z)-methoxyiminoacetamido] cephalosporins bearing 1-substituted-benzotriazol-3-ium methyl groups at the 3'-position in the cephalosporin nucleus (Scheme 1).

A general synthetic method for the preparation of $7 \beta$-[2-(2-aminothiazol-4-y1)-2(Z)-methoxyiminoacetamido]-3-[(1-substituted-benzotriazol-3-ium)methyl]3-cephem-4-carboxylate (4) is shown in Scheme 1.

1-Substituted-benzotriazole compounds (2) were prepared according to the known procedure. ${ }^{6 \sim 8)} p$ Methoxybenzyl 7 $\beta$-[2-(2-tritylaminothiazol-4-yl)-2(Z)methoxyiminoacetamido]-3-chloromethyl-3-cephem-4carboxylate (1) was readily prepared according to the previously reported method. ${ }^{2)}$ The general procedure is as follows: 1-(2-Hydroxyethyl)benzotriazole (2f, $0.20 \mathrm{~g}$, $1.2 \mathrm{mmol})$ was added to the solution of chloromethyl cephem $(1,0.78 \mathrm{~g}, 1 \mathrm{mmol})$ and $\mathrm{KI}(0.25 \mathrm{~g}, 1.5 \mathrm{mmol})$ in DMF $(1 \mathrm{ml})$ at room temperature. After being strirred at room temperature for 10 hours, the reaction mixture was poured into toluene to afford $p$-methoxybenzyl $7 \beta$-[2-(2-tritylaminothiazol-4-yl)-2( $Z$ )-methoxyiminoacetamido]-3-[(1-(2-hydroxyethyl)benzotriazol-3-ium)methyl]-3-cephem-4-carboxylate iodide (3f). Finally, the removal of all protecting groups with TFA and anisole, and then purification by column chromatography on DIAION HP-20 give $7 \beta$-[2-(2-aminothiazol-4-yl)-2(Z)methoxyiminoacetamido]-3-[(1-(2-hydroxyethyl)benzotriazol-3-ium)methyl]-3-cephem-4-carboxylate (4f) in $35.8 \%$ yield from $\mathbf{1}$. The structure of $\mathbf{4 f}$ was confirmed by ${ }^{1} \mathrm{H}$ NMR spectrum: ${ }^{1} \mathrm{H}$ NMR $(80 \mathrm{MHz}$, DMSO$\left.d_{6}+\mathrm{D}_{2} \mathrm{O}\right) \delta 3.38(\mathrm{~m}, 2 \mathrm{H}, \mathrm{C}-2), 3.88\left(\mathrm{~s}, 3 \mathrm{H},-\mathrm{OCH}_{3}\right), 4.31$ $\left(\mathrm{t}, 2 \mathrm{H},-\mathrm{CH}_{2}-\right), 4.83 \sim 5.07\left(\mathrm{~m}, 3 \mathrm{H},-\mathrm{CH}_{2}-, \mathrm{C}-7\right), 5.54$ $(\mathrm{m}, 1 \mathrm{H}, \mathrm{C}-6), 6.02(\mathrm{~d}, 2 \mathrm{H}, \mathrm{C}-3), 6.74(\mathrm{~s}, 1 \mathrm{H}$, thiazol), $7.35 \sim 8.01\left(\mathrm{~m}, 4 \mathrm{H}, \mathrm{C}_{6} \mathrm{H}_{4}\right)$.

Table 1 shows the antibacterial activity of 1 -substituted benzotriazol-3-ium cephalosporins. From examples of Table 1, 4a has the best activity against Gram-positive and Gram-negative bacteria including Pseudomonas aeruginosa, but $\mathbf{4 a}$ shows less activity than cefpirome against Streptococcus faecium MD 8B. Also 4 b has excellent activity against Gram-positive bacteria including Streptococcus faecium MD 8B and Gram-negative bacteria, and shows somewhat better activity than cefpirome and somewhat less activity than ceftazidime against $P$ seudomonas aeruginosa. With increasing the size of the benzotriazole substituent $(\mathbf{4 b} \rightarrow \mathbf{4 c} \rightarrow \mathbf{4 d})$, the activity is diminished mainly against Pseudomonas aeruginosa. This could be due to decreasing cell wall penetration. When the compound contains electron donating group like $\mathbf{4 f}$, it is still comparable to cefpirome and shows somewhat better activity than $\mathbf{4 c}$ against Gram-positive and Gram-negative bacteria. However, the compound carrying electron withdrawing group like

Scheme 1. Synthetic routes to cephalosporins.<smiles>CNc1nc(/C(=N\OC)C(=O)NC2C(=O)N3C(C(=O)O[Na])=C(CCl)CSC23)cs1</smiles>

1

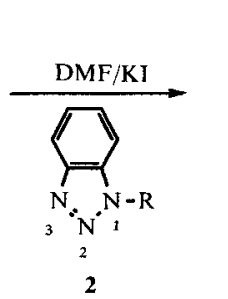<smiles>[R16]C(=O)C1=C(C[n+]2nn([R])c3ccccc32)CS[C@@H]2[C@H](NC(=O)/C(=N\OC)c3csc(NC[3H])n3)C(=O)N12</smiles>

3

TFA/anisole<smiles>[R]n1n[n+](CC2=C(C(=O)[O-])N3C(=O)C(NC(=O)/C(=N\OC)c4csc(N)n4)[C@H]3SC2)c2ccccc21</smiles> 
Table 1. Antibacterial activity of the cephalosporins 4 (MIC: $\mu \mathrm{g} / \mathrm{ml}$ ).

\begin{tabular}{|c|c|c|c|c|c|c|c|c|c|}
\hline Compound & $\mathbf{R}$ & S.p. 77A & S.f. MD88 & S.a. SG 511 & E.c. TEM & P.a. 9027 & P.a. 1592E & K.o. $1082 \mathrm{E}$ & K.a. $1512 \mathrm{E}$ \\
\hline $4 a$ & $\mathrm{NH}_{2}$ & 0.004 & 100 & 0.195 & 0.013 & 1.563 & 1.563 & 0.049 & 0.025 \\
\hline 4b & $\mathrm{CH}_{3}$ & 0.004 & 12.5 & 0.195 & 0.025 & 3.125 & 1.563 & 1.563 & 0.025 \\
\hline $4 c$ & $\mathrm{CH}_{2} \mathrm{CH}_{3}$ & 0.004 & $>100$ & 0.781 & 0.098 & 3.125 & 3.125 & 3.125 & 0.195 \\
\hline 4d & $\mathrm{CH}_{2} \mathrm{CH}=\mathrm{CH}_{2}$ & 0.007 & $>100$ & 0.781 & 0.049 & 3.125 & 6.25 & 1.563 & 0.195 \\
\hline $4 e$ & $\mathrm{CH}_{2} \mathrm{CN}$ & 0.013 & $>100$ & 1.563 & 0.025 & 3.125 & 12.5 & 3.125 & 0.391 \\
\hline $4 f$ & $\mathrm{CH}_{2} \mathrm{CH}_{2} \mathrm{OH}$ & 0.004 & $>100$ & 0.391 & 0.049 & 3.125 & 3.125 & 3.125 & 0.098 \\
\hline Ceftazidime & & 0.195 & 50 & 6.25 & 0.049 & 1.563 & 0.781 & 0.781 & 0.098 \\
\hline Cefpirome & & 0.098 & 25 & 0.391 & 0.049 & 3.125 & 3.125 & 3.125 & 0.025 \\
\hline
\end{tabular}

S.p.: Streptococcus pyogenes, S.f.: Streptococcus faecium, S.a.: Staphylococcus aureus, E.c.: Escherichia coli, P.a.: Pseudomonas aeruginosa, K.o: Klebsiella oxytoca, K.a.: Klebsiella aerogenes.

4e exhibits reduced antibacterial activity.

In summary, $\mathbf{4 a}$ and $\mathbf{4 b}$ have excellent and wellbalanced antibacterial activity against Gram-positive and Gram-negative bacteria including Pseudomonas aeruginosa, and these cephalosporins have been selected as candidates for further biological evaluation.

\section{Acknowledgment}

The financial support by Korea Ministry of Science and Technology is gratefully acknowledged.

\section{References}

1) Lattrell, R.; J. Blumbach, W. Duerckheimer, H. W. Fehlhaber, K. Fleischmann, R. Kirrstetter, B. Mencke, K.-H. ScheunemanN, E. Schrinner, W. Schwab, K. Seeger, G. Seibert \& M. Wieduwilt: Synthesis and structure-activity relationships in the cefpirome series. I. 7-[2-(2-Aminothiazol-4-yl)-2-(Z)oxyimino-acetamido]-3-[(substituted-1-pyridino)methyl]ceph-3-em-4-carboxylates. J. Antibiotics 41: 1374 1394, 1988

2) Naito, T.; S. Aburaki, H. Kamachi, Y. Narita, J. OKumura \& H. KawaguchI: Synthesis and structureactivity relationships of a new series of cephalosporins. BAY-28142 and related compounds. J. Antibiotics 39: $1092 \sim 1107,198 \dot{6}$

3) Duerckheimer, W.; J. Blumbach, R. Lattrell \& K.
H. SCheunemanN: Recent developments in the field of $\beta$-lactam antibiotics. Angew. Chem. Int. Ed. Engl. 24: $180 \sim 202,1985$

4) Mryake, A.; Y. Yoshimura, M. Yamaoka, T. Nishimura, N. Hashimoto \& A. Imada: Studies on condensedheterocyclic azolium cephalosporins. IV. Synthesis and antibacterial activity of $7 \beta$-[2-(5-amino-1,2,4-thiadiazol3-yl)-2(Z)-alkoxyiminoacetamido]-3-(condensed-heterocyclic azolium)methyl cephlaosporins including SCE2787. J. Antibiotics 45: 709 720, 1992

5) Mine, Y.; Y. Watanabe, H. SaKamoto, K. Hatano, K. Kuno, Y. Higashi, T. Kamimura, Y. Matsumoto, S. Tawara, F. Matsumoto \& S. Kuwahara: In vitro antibacterial activity of FK-037, a novel parenteral broad-spectrum cephlaosporin. J. Antibiotics 46: $71 \sim 87$, 1993

6) KATRitzky, A. R.; W. KuzMierkiewicz \& J. V. GREENHIL: An improved method for the $N$-alkylation of benzotriazole and 1,2,4-triazole. Recl. Trav. Chim. Pays-Bas 110: $369 \sim 373,1991$

7) Moon, C. J.; S. C. Park, M. G. Kim, S. H. OH, S. S. Yim, N. J. PARK, Y. K. ChOI \& M. J. Sung (Dae Woong Pharmaceutical Co., Ltd.): 3-Substututed cephem compounds. U.S. 5,336,673, Aug. 9, 1994

8) RoH, I. K.; C. J. Moon, S. C. Park, M. G. Kim, Y. K. Chol, S. H. OH, S. S. YIM, M. J. Sung \& S. G. AN (Dae Woong Pharmaceutical Co., Ltd.): Novel cephalosporins and processes for preparation thereof. WO 9221681, Dec. 10,1992 